\title{
A GENERALIZATION OF POLYNOMIAL IDENTITIES IN RINGS
}

\author{
M. P. DRAZIN
}

1. Introduction. For as long as rings and algebras have been studied for their own sakes, it has been a problem of interest to determine the consequences of various special identities and, conversely, to find sufficient conditions on a given ring which ensure that a specified identity holds; for example, the properties of being commutative, nilpotent or boundedly nil can all be defined in terms of polynomial identities. More recently, it has appeared that the existence of any nontrivial polynomial which vanishes for all choices of its arguments in a given ring $R$ has far-reaching effects on the structure of $R$. Given any ring or algebra $R$, with operators $F$, we follow the accepted course of calling $R$ a $P I$-ring or PI-algebra (i.e. "ring or algebra with a Polynomial Identity") if there is a nonzero polynomial $p\left(\lambda_{1}, \cdots, \lambda_{t}\right)$ in some finite set of noncommutative indeterminates $\lambda_{i}$, with coefficients in $F$, such that $p\left(x_{1}, \cdots, x_{t}\right)=0$ for all choices of $x_{1}, \cdots, x_{t}$ in $R$. In this note we extend known results about PI-rings to certain more general types of ring, several special cases of which have been discussed in recent literature. We assume associativity throughout.

Let $\lambda_{1}, \cdots, \lambda_{t}$ be indeterminates (sometimes referred to also as "variables"). Then, given any positive integer $d$, a monomial in $\lambda_{1}, \cdots, \lambda_{t}$ of degree $d$ will mean as usual any one of the $t^{d}$ distinct formal expressions $\pi=\lambda_{i_{1}} \cdots \lambda_{i_{d}}$ (where the suffixes $i_{1}, \cdots, i_{d}$ take independently each of the values $1, \cdots, t)$; it is convenient to write $d=d(\pi)$. Given any particular monomial $\pi=\lambda_{i_{1}} \cdots \lambda_{i_{d}}$, let $P_{\pi}$ denote the set of all monomials $\sigma$ in $\lambda_{1}, \cdots, \lambda_{t}$ (of arbitrary degree) not of the form $\lambda_{i_{1}} \cdots \lambda_{i_{h}}$ with $1 \leq h \leq d$; equivalently, $P_{\pi}$ consists of all those monomials $\sigma=\lambda_{j_{1}} \cdots \lambda_{j_{g}}$ such that either (i) $q \leq d$ and $j_{h} \neq i_{h}$ for some $h \leq q$, or (ii) $q>d$. Let $S_{\pi}$ denote the subset of $P_{\pi}$ consisting of all $\sigma \in P_{\pi}$ with $d(\sigma) \geq d(\pi)$, i.e. the set of all monomials $\sigma$ such that $d(\sigma) \geq d(\pi), \sigma \neq \pi$.

For any elements $x_{1}, \cdots, x_{t}$ of a given ring $R$ over $F$, and for any monomial $\tau=\lambda_{k_{1}} \cdots \lambda_{k_{r}}$ in $\lambda_{1}, \cdots, \lambda_{t}$, we define $\tau(x)=\tau\left(x_{1}, \cdots, x_{t}\right)$ $=x_{k_{1}} \cdots x_{k_{r}}$. Let $P_{\pi}(x)=P_{\pi}\left(x_{1}, \cdots, x_{t}\right)$ denote the subset of $R$ consisting of all $\sigma(x)$ with $\sigma \in P_{\pi}$; and define $S_{\pi}(x)=S_{\pi}\left(x_{1}, \cdots, x_{t}\right)$ analogously in the obvious way. If, for every choice of $x_{1}, \cdots, x_{t}$ in $R, \pi(x)$ lies in the right ideal of $R$ generated over $F$ by the set $P_{\pi}(x)$, then we shall say that $\pi$ is a (right) pivotal monomial for $R$ over $F$; if $\pi(x)$ always lies even in the ideal generated by the smaller set

Received by the editors October 13, 1955 and, in revised form June 6, 1956. 
$S_{\pi}(x)$, then we shall say that the monomial $\pi$ is strongly pivotal for $R$ over $F$.

In $\S \S 2$ and 3 below we establish a prima facie justification for introducing these definitions: we show that several of the more commonly considered types of ring do indeed have pivotal monomials, and that the property of having a pivotal monomial itself has striking consequences. More precisely, we prove in $\$ 2$ that every ring with minimal condition on its left or right ideals, every algebraic algebra of bounded degree, and every algebra with a polynomial identity, has a pivotal monomial; indeed, the rings in these classes each have a strongly pivotal monomial. We show also in $\$ 2$ that any pivotal monomial can always be converted into a pivotal monomial linear in each of its variables, and similarly for strongly pivotal monomials. In $\$ 3$ we prove a result essentially equivalent to the following: given any ring $R$ with a pivotal monomial, then, on writing $J$ for the Jacobson radical of $R, R / J$ is a subdirect sum of complete matrix rings of boundedly finite order over suitable division rings. Now Jacobson's structure theorem [3] states that, for any ring $R, R / J$ is a subdirect sum of primitive rings (and, conversely, that any such sum has zero radical), while the well-known Wedderburn-Artin structure theorem states that, for the special case of rings with minimal condition on right or left ideals, (i) each of the primitive subdirect summands is necessarily a complete matrix ring over a division ring, and (ii) the subdirect sum must be direct and finite. Thus, by proving (i) for the rings with pivotal monomials, we have established a half-way house between Jacobson's general theory for arbitrary rings and the Wedderburn-Artin theorem for rings with minimal condition. Also, incidentally, our result simultaneously generalizes much of Jacobson's theory of algebraic algebras of bounded degree [4].

In our final section an attempt is made to generalize the pivotal monomial concept far enough to embrace certain other interesting classes of rings. This part of the work leaves open certain questions which may prove fairly easy to answer, but some not quite trivial results are nevertheless obtained.

\section{Sufficient conditions for pivotal monomials to exist.}

THEOREM 1. If $R$ is (i) a ring with minimal condition on right ideals, or (ii) a ring with minimal condition on left ideals, ${ }^{1}$ or (iii) an algebraic algebra of bounded degree, or (iv) a PI-algebra, then $R$ has a strongly pivotal monomial.

${ }^{1}$ Since our definition of pivotality is not left-right symmetric, a result stated in terms of right ideals is of course not necessarily equivalent to the corresponding statement for left ideals. 
Proof. In cases (i), (ii) and (iii), $R$ must in fact have a pivotal monomial $\lambda^{m}$ in a single variable $\lambda$, i.e. a positive integer $m$ exists such that, for each $x \in R$, there is a corresponding element $a \in R$ satisfying $x^{m}=x^{m+1} a$ (and clearly a pivotal monomial in a single variable is always strongly pivotal). This has been pointed out by Arens and Kaplansky [1, Theorem 3.1] in case (i); also (e.g. by a remark of Kaplansky [6, p. 74]) the element $a$ can always be chosen so as to commute with $x$, whence (by a legitimate application of left-right symmetry) we deduce the result in case (ii). The case (iii) is trivial: for, if each element $x$ of a ring $R$ satisfies a polynomial equation (over some given field $F$ ) of degree not exceeding a given positive integer $m$, then each $x^{m}$ is expressible as a linear combination of higher powers.

We have now only to deal with case (iv). Any polynomial identity of a ring $R$ is expressible in the form $\sum_{k=0}^{u} \alpha_{k} \sigma_{k}(x)=0$, where $u$ is some non-negative integer, the $\alpha_{k}$ are nonzero elements of the operator ring $F$, and the $\sigma_{k}$ are distinct monomials in some finite set of variables $\lambda_{1}, \cdots, \lambda_{t}$. If $R$ is an algebra, i.e. if $F$ is a field, then, selecting a monomial of minimal degree from the set $\sigma_{0}, \cdots, \sigma_{u}$, say $\sigma_{0}$, we have $\sigma_{0}(x)=-\sum_{k=1}^{u} \alpha_{0}^{-1} \alpha_{k} \sigma_{k}(x)$ identically; equivalently, $\pi(x)=\sum_{k=1}^{u} \beta_{k} \sigma_{k}(x)$, where each $\beta_{k}=-\alpha_{0}^{-1} \alpha_{k} \in F$ and $\pi\left(=\sigma_{0}\right)$, $\sigma_{1}, \cdots, \sigma_{u}$ are distinct monomials with $d(\pi) \leq d\left(\sigma_{k}\right)(k=1, \cdots, u)$, so that clearly each $\sigma_{k} \in S_{\pi}$. Thus, for arbitrary $x_{1}, \cdots, x_{t} \in R$, we have $\sigma_{k}(x) \in S_{\pi}(x)(k=1, \cdots, u)$, and so $\pi(x)$ lies in the submodule of $R$ generated by $S_{\pi}(x)$. In particular, $\pi$ is strongly pivotal for $R$, as required.

Wagner [11, Satz 2] and later Kaplansky [5, Lemma 1] have pointed out that every PI-algebra must in fact satisfy an identity in only two variables; and it is easy, by using their device of replacing $\lambda_{i}$ by $\lambda^{i-1} \mu(i=1, \cdots, t)$, to prove the analogous result for rings with pivotal monomials. Less trivially, we have the following analogues of Kaplansky's other result (Lemma 2) that the existence of a polynomial identity implies that of an identity in which each variable enters linearly:

Theorem 2. (i) If $R$ has a pivotal monomial, then it has a pivotal monomial linear in each variable; (ii) if $T$ has a strongly pivotal monomial, then it has a strongly pivotal monomial linear in each variable.

Proof. (i) Let $\pi=\lambda_{i_{1}} \cdots \lambda_{i_{d}}$ be pivotal for $R$ with respect to some given finite set of variables $\lambda_{1}, \cdots, \lambda_{t}$. If any $\lambda_{i}$, say $\lambda_{1}$, occurs in $\pi$ more than once, say $r$ times, we shall show how to obtain a new 
pivotal monomial $\pi_{1}$, in $t+1$ variables $\lambda, \mu, \lambda_{2}, \cdots, \lambda_{t}$, of degree $r-1$ in $\lambda$, linear in $\mu$, and of the same degree as $\pi$ in each of $\lambda_{2}, \cdots, \lambda_{t}$. By repeating this process enough times we obtain a pivotal monomial for $R$, of the same total degree as $\pi$, and linear in each of its variables (whose number may have been increased to $t+d-1)$.

For any monomial $\tau$ in $\lambda_{1}, \cdots, \lambda_{t}$, the set of all monomials in $\lambda, \mu, \lambda_{2}, \cdots, \lambda_{t}$ which become identical with $\tau$ under the substitutions $\lambda \rightarrow \lambda_{1}, \mu \rightarrow \lambda_{1}$, is clearly finite; indeed, if $\lambda_{1}$ enters into $\tau$ with degree $s$, this set will contain precisely $2^{s}$ distinct monomials, which we shall denote by $\tau_{1}, \cdots, \tau_{2^{s}}$. Then $d\left(\tau_{j}\right)=d(\tau)$ for each $j$, and, for any $x, y, x_{2}, \cdots, x_{t} \in R$, we have

$$
\tau\left(x+y, x_{2}, \cdots, x_{t}\right)=\sum_{j=1}^{2^{s}} \tau_{j}\left(x, y, x_{2}, \cdots, x_{t}\right) .
$$

We apply this first with $\tau=\pi$. Since $\lambda_{1}$ occurs $r$ times in $\pi$, the set of $\pi_{j}$ includes $r$ members linear in $\mu$; choose any one of these, and renumber the remaining $\pi_{j}$ so that this selected monomial is $\pi_{1}$. Now, for any $\sigma\left(\lambda_{1}, \cdots, \lambda_{t}\right) \in P_{\pi}$, we notice on taking $\tau=\sigma$ that all the $\sigma_{j}\left(\lambda, \mu, \lambda_{2}, \cdots, \lambda_{t}\right)$ are in $P_{\pi_{1}}$ (for if some monomial $\sigma_{j}$ could be obtained by curtailing $\pi_{1}\left(\lambda, \mu, \lambda_{2}, \cdots, \lambda_{t}\right)$ at some point, then $\sigma\left(\lambda_{1}, \lambda_{2}, \cdots, \lambda_{t}\right)$ could be obtained by curtailing $\pi\left(\lambda_{1}, \lambda_{2}, \cdots, \lambda_{t}\right)$ at the corresponding point, contrary to our supposition that $\sigma \in P_{\boldsymbol{\pi}}$ ). Since $\pi$ is pivotal for $R$ in $\lambda_{1}, \cdots, \lambda_{t}$, therefore, for any $x, y, x_{2}, \cdots$, $x_{t} \in R$, of course $\pi\left(x+y, x_{2}, \cdots, x_{t}\right)$ is in the right ideal of $R$ generated by those $\sigma\left(x+y, x_{2}, \cdots, x_{t}\right)$ with $\sigma \in P_{\pi}$; in view of $\left(^{*}\right)$ with $\tau=\sigma$, it follows that $\pi\left(x+y, x_{2}, \cdots, x_{t}\right)$ is in the ideal generated by $P_{\pi_{1}}\left(x, y, x_{2}, \cdots, x_{t}\right)$. But, by $\left(^{*}\right)$ with $\tau=\pi$, we have

$$
\pi_{1}\left(x, y, x_{2}, \cdots, x_{t}\right) \quad \pi\left(x+y, x_{2}, \cdots, x_{t}\right)-\sum_{j=2}^{2^{r}} \pi_{j}\left(x, y, x_{2}, \cdots, x_{t}\right),
$$

and each $\pi_{j}\left(\lambda, \mu, \lambda_{2}, \cdots, \lambda_{t}\right)$ with $j \geq 2$ is obviously in $P_{\pi_{1}}$ (since each has the same degree as $\pi_{1}$, and none is identical with $\pi_{1}$ ). Thus, for any $x, y, x_{2}, \cdots, x_{t} \in R$, we see that $\pi_{1}\left(x, y, x_{2}, \cdots, x_{t}\right)$ is in the right ideal of $R$ generated by $P_{\pi_{1}}\left(x, y, x_{2}, \cdots, x_{t}\right)$, i.e. $\pi_{1}$ is pivotal.

The result (i) now follows at once by induction on the sum of the degrees of those $\lambda_{i}$ that occur nonlinearly in $\pi$.

(ii) If $\pi$ is given to be strongly pivotal then (since in the argument above $d(\pi)=d\left(\pi_{1}\right)$ and we did not introduce any $\sigma$ 's to "generate" $\pi_{1}\left(x, y, x_{2}, \cdots, x_{t}\right)$ having lower total degree than those necessary 
to generate $\left.\pi\left(x+y, x_{2}, \cdots, x_{t}\right)\right)$ clearly $\pi_{1}$ is also strongly pivotal; the induction then proceeds as for (i), and the theorem is proved.

Thus, by (i), if $R$ has a pivotal monomial $\pi$ of degree $d$, then, by suitably renumbering the given $\lambda_{i}$ and (possibly) adjoining some new ones, we may take $\pi$ in the form $\pi=\lambda_{1} \lambda_{2} \cdots \lambda_{d}$; and we may of course suppose also that $t=d$ (for if $t>d$ we should find, on taking $x_{d+1}=\ldots$ $=x_{t}=0$ in the definition of pivotality, that $\lambda_{d+1}, \cdots, \lambda_{t}$ were not needed). In other words, the existence of any pivotal monomial for $R$, say of degree $d$, simply means that, for any $x_{1}, \cdots, x_{d}$ in $R$, the product $x_{1} \cdots x_{d}$ is in the right ideal of $R$ generated by the $d(d-1)$ products $x_{1} x_{2} \cdots x_{i-1} x_{j}(i, j=1, \cdots, d ; i \neq j)$ together with the $d$ products $x_{1} \cdots x_{d} x_{j}(j=1, \cdots, d)$.

Kaplansky [5, Theorem 5] used a result analogous to Theorem 2 to show that every nil PI-algebra is locally finite (or, equivalently, locally nilpotent). However, his arguments do not seem to extend to the more general case of rings with pivotal monomials. If $R$ has a strongly pivotal monomial $\pi$, then Theorem 2 (ii) shows that we can again take $\pi$ in the form $\pi=\lambda_{1} \lambda_{2} \cdots \lambda_{d}$ with $t=d$. In other words, for any $x_{1}, \cdots, x_{d} \in R$, the product $x_{1} \cdots x_{d}$ is in the right ideal of $R$ generated by the $d$ products $x_{1} x_{2} \cdots x_{d} x_{j}$ and all the $d$-ary products $x_{k_{1}} \cdots x_{k_{d}}$ other than $x_{1} \cdots x_{d}$ itself. If we could restrict $k_{1}, \cdots, k_{d}$ to the $d !-1$ nontrivial permutations of $1, \cdots, d$, we could then argue in the same way as Kaplansky; but, even in the nil case, there seems to be no easy way of dealing with rings in which, say, $x y$ is always contained in the right ideal generated by $x^{2}, y x$ and $y^{2}$.

These difficulties can, however, be overcome by imposing the stronger hypothesis that $\pi(x)$ lies in the module generated over $F$ by $\sigma(x)$ 's with the $\sigma$ 's restricted to some prescribed finite subset of $P_{\pi}$ (independent of $x_{1}, \cdots, x_{t}$ ). This is a consequence of Kaplansky's Theorem 5 itself and the following generalization, pointed out to the writer by Dr. Graham Higman, of Kaplansky's Theorem 3 (which states that every algebraic algebra of bounded degree is a PI-algebra):

TheORem 3. Let $\sigma_{1}, \cdots, \sigma_{u}$ be any finite set of distinct monomials in $\lambda_{1}, \cdots, \lambda_{t}$. Then there is a nonzero polynomial $p\left(\lambda_{0}, \lambda_{1}, \cdots, \lambda_{t}\right)$, homogeneous in each of its arguments and with each nonzero coefficient \pm 1 , such that any relation of the form $\sum_{j=1}^{u} \alpha_{j} \sigma_{j}(x)=0$, for given elements $\alpha_{1}, \cdots, \alpha_{u}$ of $F$ and a given set $(x)=\left(x_{1}, \cdots, x_{t}\right)$ of elements of a given ring $R$ over $F$, implies, for all $x_{0} \in R$,

$$
\alpha, p\left(x_{0}, x_{1}, \cdots, x_{t}\right)=0 \quad(j=1, \cdots, u) .
$$

Proof. Higman's own argument was along the same lines as Kaplansky's. However, the result can be obtained more easily in terms of 
the "standard polynomial" $s\left(\mu_{1}, \cdots, \mu_{u}\right)=\sum \pm \mu_{i_{1}} \cdots \mu_{i_{u}}$, this sum being extended over all the $u$ ! distinct permutations $i_{1}, \cdots, i_{u}$ of $1, \cdots, u$, the typical summand $\mu_{i_{1}} \cdots \mu_{i_{u}}$ being allocated a plus or minus sign according as the corresponding permutation is even or odd. $^{2}$

We note first that, for any $y_{1}, \cdots, y_{u} \in R$ satisfying $\sum_{j=1}^{u} \alpha_{j} y_{j}=0$, we have

$$
\begin{aligned}
\alpha_{1} s\left(y_{1}, \cdots, y_{u}\right) & =s\left(\alpha_{1} y_{1}, y_{2}, \cdots, y_{u}\right)=s\left(-\sum_{j=2}^{u} \alpha_{j} y_{j}, y_{2}, \cdots, y_{u}\right) \\
& =-\sum_{j=2}^{u} \alpha_{j} s\left(y_{j}, y_{2}, \cdots, y_{u}\right)=0,
\end{aligned}
$$

since of course $s$ vanishes whenever a pair of its arguments are taken equal. Thus, if we define

$$
p\left(\lambda_{0}, \lambda_{1}, \cdots, \lambda_{t}\right)=s\left(\lambda_{0} \sigma_{1}, \lambda_{0} \sigma_{2}, \cdots, \lambda_{0} \sigma_{u}\right),
$$

then $\sum_{j=1}^{u} \alpha_{j} \sigma_{j}(x)=0$ implies that $\alpha_{1} p\left(x_{0}, x_{1}, \cdots, x_{t}\right)=0$ for all $x_{0} \in R$, and, similarly, that $\alpha_{\jmath} p\left(x_{0}, x_{1}, \cdots, x_{t}\right)=0(j=2, \cdots, u)$. Finally, clearly this choice of $p\left(\lambda_{0}, \lambda_{1}, \cdots, \lambda_{t}\right)$ is nonzero and satisfies the other stated conditions.

3. Primitive rings with pivotal monomials. Kaplansky [5, Theorem $1]$ showed that every primitive PI-algebra $R$ is finite-dimensional over its centre. His proof depends on showing first that $R$ is isomorphic with a complete matrix ring over a division ring (which must clearly satisfy any identity satisfied by $R$, so that it is then sufficient to consider the case in which $R$ is itself a division ring). We cannot hope to prove for primitive rings with strongly pivotal monomials the analogue of Kaplansky's full result (which analogue would entail that, contrary to known examples, every division ring is finite-dimensional over its centre), but the analogue of Kaplansky's preliminary result does hold. Indeed, more generally,

THEOREM 4. The following statements about an arbitrary primitive ring $R$ are equivalent: (i) $R$ has a pivotal monomial; (ii) $R$ is isomorphic with a complete ring $M_{q}(D)$ of $q \times q$ matrices over a division ring $D$; (iii) $R$ has a (necessarily strongly) pivotal monomial $\lambda^{m}$ in a single variable $\lambda$.

2 Polynomials of this type were, apparently, first considered by Kolchin (see [8]) and F. W. Levi [9]; cf. also [11]. The argument which follows generalizes that of Amitsur in [10, Theorem 5]. 
Proof. The equivalence of (ii) and (iii) has been stated by Arens and Kaplansky [1, Theorem 3.1], but our proof is self-contained. Since (iii) implies (i) trivially, it will be enough to show that (i) implies (ii) and that (ii) implies (iii). Our proof that (i) implies (ii) is essentially a generalized and precise formulation of a technique which has by now become standard, but which has hitherto (see for example $[2$, Theorem $2.2 ; 7]$ ) been applied only in special cases. ${ }^{3}$

Suppose then that (i) holds, and let $\pi=\lambda_{i_{1}} \cdots \lambda_{i_{d}}$ be a pivotal monomial in variables $\lambda_{1}, \cdots, \lambda_{t}$. Being primitive, $R$ may be represented as a dense ring of linear transformations of a vector space $V$ over a division ring $D$; we may regard $R$ and $D$ as operating on $V$ from the right, and we have to show that $V$ is finite-dimensional over $D$. In fact $V$ must have dimension $d$ at most. For, given any $d+1$ $D$-independent elements $v_{0}, v_{1}, \cdots, v_{d}$ of $V$, we could choose $x_{1}, \cdots, x_{t}$ in $R$ so that, for each $i=1, \cdots, t$,

$$
v_{h-1} x_{i}=\delta_{i_{h}}^{i} v_{h} \quad(h=1, \cdots, d), \quad v_{d} x_{i}=0
$$

(where $\delta$ is Kronecker's symbol, i.e. $\delta_{j}^{i}=1$ if $i=j$ and is otherwise zero). Then evidently $v_{0} \pi(x)=v_{d}$, while, for any $\sigma \in P_{\boldsymbol{x}}, v_{0} \sigma(x)=0$. Since $\pi(x)$ is in the right ideal of $R$ generated by these $\sigma(x)$, it would follow that $v_{d}=0$, contrary to our supposition that $v_{0}, \cdots, v_{d}$ were $D$-independent. Hence $V$ cannot contain more than $d$ independent elements, i.e. (ii) holds with $q \leq d$.

To prove that (ii) implies (iii), we note that the complete ring $M_{q}(D)$ of $q \times q$ matrices over a division ring $D$ is a right vector space over $D$ of finite dimension $q^{2}$, so that every element of $M_{q}(D)$ satisfies a nontrivial polynomial equation over $D$, possibly with constant term, of degree $q^{2}$ (and, by the Cayley-Hamilton theorem, ever of degree $q$ if $D$ happens to be commutative). Hence, for any $x \in M_{q}(D)$, we can find $a \in M_{q}(D)$ so that $x^{q^{2}}=x^{q^{2}+1} a$, i.e. $\lambda^{q^{2}}$ is a pivotal monomial for $M_{q}(D)$. (In fact so is $\lambda^{q}$, even for noncommutative $D$, but we shall not stop to prove this here.)

We remark in passing that, if each $\pi(x)$ is even in the ideal generated by those $\sigma(x)$ with $\sigma$ satisfying the first of the defining conditions of pivotality (as will always be the case if $R$ satisfies a homogeneous polynomial identity such as $\left.(x y-y x)^{2}(x-y)=0\right)$, then we have in (ii) the strict inequality $q<d(\pi)$ : to see this one has only to drop $v_{d}$ from the proof above, and replace the transformation laws imposed there by

8 It is relevant to affirm here that, in spite of the report on [2] in the Mathematical Reviews vol. 17 (1956) p. 1048, the majority of the results in [2], and in particular Theorem 2.2, are indeed correct; cf. [2a]. 


$$
v_{h-1} x_{i}=\delta_{i_{h} v_{h}}^{i} \quad(h=1, \cdots, d-1), \quad v_{d-1} x_{i}=\delta_{i_{d}}^{i} v_{d-1} .
$$

Again, in connexion with the remarks immediately preceding Theorem 3 above, we can (without any appeal to Theorem 3) prove

THEOREM 5. Let $R$ be a given primitive ring and $\pi$ any monomial in $\lambda_{1}, \cdots, \lambda_{t}$, and suppose that, for all choices of $x_{1}, \cdots, x_{t}$ in $R, \pi(x)$ is in the submodule of $R$ generated by ALL the $\sigma(x)$ with $\sigma \neq \pi$. Then $R$ satisfies the conditions of Theorem 4.

To show this, one has only to apply the argument used above to deduce (ii) from (i): we no longer get $v_{d}=0$, but we do at any rate find that $v_{d}$ is a linear combination of $v_{0}, \cdots, v_{d-1}$, which is still enough to give the required contradiction. The writer is indebted to Professor P. Hall and Mr. D. Rees for this observation.

4. Pivotal sets. Besides the rings with pivotal monomials, there are other types of ring for which primitivity is known to imply the property of being a complete matrix ring (or something still stronger). For example, a primitive nil ring can only contain the single element 0 ; more generally (cf. [2, Theorem 6.3]), if to each pair $x, y$ of elements of a given primitive ring $R$ there correspond positive integers $m, s$ such that $x^{m} y^{s}$ lies in the right ideal generated by $y$, then $R$ must be a division ring. We now seek to extend Theorem 4 far enough to cover such cases.

To this end, we consider "pivotal sets" of monomials. Given any set $\Pi$ of monomials in $\lambda_{1}, \cdots, \lambda_{t}$, define $P_{\mathrm{II}}=\bigcap_{\pi \in \mathrm{I}} P_{\pi}$; equivalently, $P_{\mathrm{II}}$ is the complement, in the set of all monomials in $\lambda_{1}, \cdots, \lambda_{t}$, of that subset obtainable from members of $\Pi$ by curtailment. For any $x_{1}, \cdots, x_{t}$ in a ring $R$, let $P_{\mathbf{\Pi}}(x)$ denote the set of all $\sigma(x)$ with $\sigma \in P_{\mathbf{\Pi}}$. Then we shall call $\Pi$ a pivotal set of monomials for $R$ in $\lambda_{1}, \cdots, \lambda_{t}$ if, to each choice of $x_{1}, \cdots, x_{t}$ in $R$, there corresponds some $\pi \in \Pi$ (not necessarily the same $\pi$ for each choice of the $x$ 's) such that $\pi(x)$ lies in the right ideal generated by $P_{\mathbf{I}}(x)$; if $P_{\mathbf{I}}$ is empty we interpret this as meaning $\pi(x)=0$ (so that $R$ must in fact be nil). This definition is consistent with our previous one if $\Pi$ contains only a single member $\pi$. Clearly, for a nil ring, the set of all monomials in $\lambda_{1}, \cdots, \lambda_{t}$ is pivotal.

Unfortunately the argument of Theorem 4 does not extend to rings with arbitrary pivotal sets; indeed, the existence of any such extension seems implausible (though the writer knows no counterexample). It is not hard to extend Theorem 4 to cover the case of finite pivotal sets $\Pi$. However, this generalization is not a significant one when the monomials in $\Pi$ arise from polynomial identities in the way described in the proof of Theorem 1: for, if $p_{k}\left(\lambda_{1}, \cdots, \lambda_{t}\right)$ 
$(k=1, \cdots, n)$ is any given finite set of nonzero polynomials over a given field $F$, and if for each choice of $x_{1}, \cdots, x_{t}$ in a given ring $R$ over $F$ at least one of the $p_{k}\left(x_{1}, \cdots, x_{t}\right)$ vanishes, then $R$ is a PIalgebra relative to the (nonzero) polynomial $p_{1}\left(\lambda_{1}, \cdots, \lambda_{t}\right) \cdots$ $p_{n}\left(\lambda_{1}, \cdots, \lambda_{t}\right)$, and so Theorem 4 already applies. The generalization does nevertheless have some interest for rings with finite pivotal sets not necessarily arising from polynomial identities, but it is not broad enough to cover the nil rings.

We overcome this difficulty by introducing the idea of "nested sets." Given any two monomials $\pi_{1}, \pi_{2}$ in the same set of variables $\lambda_{1}, \cdots, \lambda_{t}$, we denote by $\pi_{1} \pi_{2}$ the product monomial (of degree $\left.d\left(\pi_{1} \pi_{2}\right)=d\left(\pi_{1}\right)+d\left(\pi_{2}\right)\right)$ formed by writing down the successive terms of $\pi_{1}$, followed by those of $\pi_{2}$. Next, given any two sets $\Pi_{1}, \Pi_{2}$ of monomials in $\lambda_{1}, \cdots, \lambda_{t}$, let $\Pi_{1} \Pi_{2}$ denote the product set consisting of all monomials of the form $\pi_{1} \pi_{2}$ with $\pi_{1} \in \Pi_{1}, \pi_{2} \in \Pi_{2}$; and define products of any finite number of sets in the natural way. For each $i=1, \cdots, t$, let $\Phi_{i 1}$ denote the set consisting of $\lambda_{i}$ alone, and let $\Phi_{i 2}$ denote the set of all $\lambda_{i}^{r}(r=1,2, \cdots)$; for example, the sets $\Phi_{12}$, $\Phi_{12} \Phi_{22}$ are respectively pivotal for nil rings and for the other type of ring mentioned at the beginning of this section. Then any set of the form $\Pi=\Phi_{i_{1} j_{1}} \cdots \Phi_{i_{u} j_{u}}$ (where $u$ can be any positive integer, and the $i_{r}, j_{r}$ satisfy $\left.1 \leq i_{r} \leq t, 1 \leq j_{r} \leq 2\right)$ will be called a nested set of monomials in $\lambda_{1}, \cdots, \lambda_{t}$; clearly such a set $\Pi$ will be finite if and only if $j_{1}=\ldots$ $=j_{u}=1$, in which case II reduces to the single monomial $\lambda_{i_{1}} \cdots \lambda_{i_{u}}$.

THEOREM 6. Every primitive ring with a nested pivotal set of monomials is isomorphic with a complete ring $M_{q}(D)$ of matrices over a division ring $D$.

Proof. Let $\Pi=\Phi_{i_{1} j_{1}} \cdots \Phi_{i_{u} j_{u}}$ be a given nested pivotal set of monomials for a given primitive ring $R$; we may suppose without loss of generality that, if any given pair $i_{h-1}, i_{h}$ of consecutive suffixes are equal, then $j_{h-1}=1$. We shall obtain the result, with $q \leq u$, by showing that $V$ (defined as in the proof of Theorem 4) cannot contain $u+1 D$-independent elements $v_{0}, v_{1}, \cdots, v_{u}$. For, if such elements existed, then, for each $i=1, \cdots, t$, we could choose $x_{i}$ in $R$ such that $v_{0} x_{i}=\delta_{i_{1}}^{i} v_{1}, v_{h-1} x_{i}=\delta_{i_{h}}^{i} v_{h}+\delta_{i_{h-1}}^{i} \delta_{h_{h-1}}^{2} v_{h-1} \quad(h=2, \cdots, u), \quad v_{u} x_{i}=\delta_{i_{u}}^{i} \delta_{j_{u}}^{2} v_{u}$. Then, for any $\pi \in \Pi$, we should again find that $v_{0} \pi(x)=v_{u}$, while $v_{0} \sigma(x)=0$ for every $\sigma \in P_{\mathrm{II}}$. Since $\Pi$ is pivotal for $R$, we should then have $v_{u}=0$, contrary to our supposition of $D$-independence. This contradiction establishes the theorem.

In the case $\Pi=\Phi_{11}^{m} \Phi_{22}$, where $m$ is any given positive integer, the above proof reduces to that given in [2, Theorem 2.2]. In some special 
cases (e.g. for nil rings and for the other type of ring described at the beginning of this Section) the upper bound $u$ for $q$ can be improved. However, no such refinement is needed to deduce

THEOREM 7. Let $R$ be a given ring with a nested pivotal set of monomials, and let $J$ denote the Jacobson radical of $R$. Then $R / J$ is isomorphic with a subdirect sum of complete matrix rings (of bounded order) over division rings.

This follows from Jacobson's structure theory [3], the proof of Theorem 6 above, and the evident fact that a pivotal set for a ring $R$ is necessarily pivotal for every homomorphic image of $R$.

It is not difficult to extend Theorem 6 (and hence also Theorem 7) to more general pivotal sets. One could, for example, cope with products $\Pi$ including also factors $\Phi_{i 3}$ consisting of just $\lambda_{i}$ and the empty monomial, or factors $\Phi_{i 4}$ consisting of all $\lambda_{i}^{r}(r=1,2, \cdots)$ together with the empty monomial; but even such a result, though of somewhat greater scope, would presumably not be best possible in any real sense, and so we have preferred to restrict our discussion to one simple representative case.

\section{REFERENCES}

1. R. Arens and I. Kaplansky, Topological representation of algebras, Trans. Amer. Math. Soc. vol. 63 (1948) pp. 457-481.

2. M. P. Drazin, Engel rings and a result of Herstein and Kaplansky, Amer. J. Math. vol. 77 (1955) pp. 895-913.

2a. - Corrections to [2], Amer. J. Math. vol. 78 (1956) p. 899.

3. N. Jacobson, The radical and semi-simplicity for arbitrary rings, Amer. J. Math. vol. 67 (1945) pp. 300-320.

4. - Structure theory for algebraic algebras of bounded degree, Ann. of Math. (2) vol. 46 (1945) pp. 695-707.

5. I. Kaplansky, Rings with a polynomial identity, Bull. Amer. Math. Soc. vol. 54 (1948) pp. 575-580.

6. - Topological representation of algebras II, Trans. Amer. Math. Soc. vol. 68. (1950) pp. 62-75.

7. - A theorem on division rings, Canadian Journal of Mathematics vol. 3 (1951) pp. 290-292.

8. - Groups with representations of bounded degree, Canadian Journal of Mathematics vol. 1 (1949) pp. 105-112.

9. F. W. Levi, Über den Kommutativitätsvang in einem Ringe, Math. Ann. vol. 121 (1949) pp. 184-190.

10. S. A. Amitsur, Identities and generators of matrix rings, Bull. Res. Council Israel (A) vol. 5 (1955) pp. 5-10.

11. W. Wagner, Über die Grundlagen der projektiven Geometrie und allgєmeine Zahlensysteme, Math. Ann. vol. 113 (1937) pp. 528-567.

Trinity College, Cambridge 